\title{
BMJ Open Study protocol to examine the relationship between environmental exposure to lead and blood lead levels among children from day-care centres in Ekurhuleni Metropolitan Municipality
}

\author{
Mbalenhle Desiree Cindi, ${ }^{1}$ Thokozani Patrick Mbonane (i) , ${ }^{1}$ Nisha Naicker (D) ${ }^{1,2,3}$
}

To cite: Cindi MD, Mbonane TP, Naicker N. Study protocol to examine the relationship between environmental exposure to lead and blood lead levels among children from day-care centres in Ekurhuleni Metropolitan Municipality. BMJ Open 2020;10:e036687. doi:10.1136/ bmjopen-2019-036687

- Prepublication history for this paper is available online. To view these files, please visit the journal online (http://dx.doi. org/10.1136/bmjopen-2019036687).

Received 27 December 2019

Revised 06 April 2020 Accepted 06 May 2020

Check for updates

(C) Author(s) (or their employer(s)) 2020. Re-use permitted under CC BY-NC. No commercial re-use. See rights and permissions. Published by BMJ.

${ }^{1}$ Department of Environmental Health, Faculty of Health Sciences, University of Johannesburg, Auckland Park, Gauteng, South Africa ${ }^{2}$ Epidemiology and Surveillance Section, National Institute of Occupational Health, Johannesburg, Gauteng, South Africa

${ }^{3}$ School of Public Health, Faculty of Health Sciences, University of witwatersrand, Johannesburg, Gauteng, South Africa

Correspondence to

Mr Thokozani Patrick Mbonane; tmbonane@uj.ac.za

\section{ABSTRACT}

Introduction Lead exposure is toxic to all humans and is very harmful to young children, especially 5 -year-olds. Elevated blood lead levels (BLLs) in children have been associated with their daily surrounding environment. This protocol seeks to evaluate the association between environmental lead exposure and BLLs among children in day-care centres, including household and other risk factors.

Methods and analysis To achieve the objectives of the study, we adopted a cross-sectional analytical design. A portable X-ray fluorescence analyser was used for environmental sampling, and BLLs were determined using the LeadCare II machine among preschool children. Household and other risk factors were assessed using a questionnaire. Random sampling was employed to select day-care centres in the municipality and children in each day-care centre. Data will be analysed using SPSS V. 26. Ethics and dissemination Ethical approval and permission were obtained prior to commencement of the study. The researcher intends to publish the results in peer-reviewed journals and also to present a paper at a scientific conference. The study will generate information on environmental lead exposure among vulnerable children (2-5 years), and it will promote public health action to prevent long-term exposure in day-care centres.

\section{INTRODUCTION}

Lead $(\mathrm{Pb})$ contamination in children's environment is a global public health concern, especially in poor communities and countries. ${ }^{1} 2$ Low-to-middle-income countries (LMICs) have a higher economic burden of childhood lead exposure when compared with rich countries. ${ }^{3}$ However, there is limited or non-existent public health intervention in poor countries. Recent studies have shown an association between environmental lead exposure and elevated blood lead levels (BLLs) and children's environment.

Most children in LMICs are exposed to lead due to the failure to adopt and implement legislation that promotes a lead-free
Strengths and limitations of this study:

- The study is the first to determine a relationship between blood lead levels and environmental lead exposure as well as possible household exposure among preschool children in a low-to-middleincome country (LMIC).

- The instruments for blood and environmental sampling have been used and validated in LMICs; this ensured study validity and reliability.

- The self-administered questionnaire could introduce response bias.

- The use of random sampling ensures minimisation of selection bias

- The study used screening tools for data collection; this could be a study limitation.

environment. ${ }^{4}$ Children in South African cities ingest lead from contaminated soil, peeling paint chips from walls, toys and water due to rusted steel pipes. ${ }^{5}$ Eating foods cooked using recycled aluminium pots is an emerging risk, but it is not yet understood. ${ }^{6}$ Inhalation of lead-contaminated air due to proximity to non-rehabilitated mine dumps and household dust is also common. In areas such as Ekurhuleni Metropolitan Municipality, numerous sources (such as leaded paint, illegal gold mining, running a business in a backyard in a residential area, especially a steel-recycling business, residing in an old house and living close to lead-emitting industries) contribute to daily environmental lead exposure. $^{5}$

Childhood lead risk factors differ with age group and period and level of exposure. ${ }^{7}$ Children are the most vulnerable population group and are affected by low to high environmental lead exposure. Severe lead intoxication might affect children due to hand-to-mouth habits, and in children lead 
is absorbed in the small intestine more easily than in adults, as children are still developing. ${ }^{8}$ Elevated BLLs have been linked to developmental delays, which may have other health implications for the child. A study conducted among Taiwan children found that low lead levels contributed to developmental delays in young children after controlling their health status. ${ }^{9}$ Exposure to lead on a daily basis in young children can lead to the development of learning difficulties, short concentration span, emotional problems, antisocial behaviour, poor academic performance and neurodevelopmental deficits. ${ }^{10-15}$ These conditions, especially neurodevelopmental effects, may cause exposed children to engage in delinquent behaviour, aggression, violent behaviour or criminal behaviour in their later years. ${ }^{16-19}$

Studies conducted in high-income countries show that children from these countries have lower BLLs when compared with LMICs. ${ }^{20}{ }^{21}$ An observational study conducted among children at risk of lead exposure in the USA found that $20 \%$ of children studied had BLLs below $10 \mu \mathrm{g} / \mathrm{dL} .{ }^{20}$ Yet, these BLLs had an impact on the children's reading readiness. A study conducted in China found that $8 \%$ of preschool children aged 3-5 years had BLLs above $10 \mu \mathrm{g} / \mathrm{dL} .{ }^{1}$ A cross-sectional study in a city in Botswana found that $5 \%$ of children aged 1-6 years had BLLs higher than $10 \mu \mathrm{g} / \mathrm{dL} .{ }^{22}$ However, these studies did not determine lead contamination in children's physical learning environment; they focused only on the household, parental and community risk factors.

African children and children in other regions, such as Asia, have spent most of their time in a lead-contaminated environment. ${ }^{23-25}$ Even in South Africa, observational studies have found lead contamination in the environment where children play, study and live. ${ }^{26-29}$ Mathee and colleagues found that equipment in the children's parks of three Gauteng Metropolitan Municipalities was highly contaminated with lead. ${ }^{26}$ Another study found high lead levels in the residential gardens of four suburbs in Johannesburg. ${ }^{28}$ Despite these previous findings, there are no studies that have assessed BLLs among toddlers or children under 5 years in South Africa and other LMICs in the sub-Saharan region. Yet, concerns have been raised about this age group, due to their tendency of hand-to-mouth habits. It is important to understand BLLs and lead levels in children's environment, in order to develop, promote and implement appropriate public health prevention strategies.

There is growing evidence of the link between BLLs and children's environment in high-income countries and a few LMICs, including South Africa. There are no scientifically proven safe levels of environmental lead exposure for either the short term or the long term in children and adults. ${ }^{30}$ Yet, there is no baseline for BLLs for 5 -year-olds in most LMICs. In this study, we assume that children under the age of 5 years are still exposed to high environmental lead exposure in households, in entertainment areas and at preschool. Thus, we aimed to evaluate the association between environmental lead exposure, BLLs and other risk factors among day-care children in Ekurhuleni Metropolitan Municipality. The following objectives were formulated to achieve the aim of the study:

- To determine the lead concentrations in selected objects found and used in day-care centres in Ekurhuleni Metropolitan Municipality.

- To measure the BLLs in children aged between 2 and 5 years in Ekurhuleni Metropolitan Municipality daycare centres.

- To determine the household, parental and other risk factors that might be associated with elevated BLLs.

- To assess the association between preschool environment lead exposure, BLLs and household risk factors after controlling for confounders.

\section{METHODS AND ANALYSIS}

The study adopted a cross-sectional analytical design to evaluate the level of childhood exposure to lead, by assessing environmental lead exposure and BLLs and in day-care centres situated in Ekurhuleni Metropolitan Municipality. This study commenced at the beginning of 2019 and was anticipated to be completed at the end of April 2020.

\section{Population, sampling and sample size}

Ekurhuleni Metropolitan Municipality has a total of 263 day-care centres that are registered with the Gauteng Department of Health, with a total of 9217 children aged between 0 and 5 years. The study was conducted in the three sub-district regions (East, North and South) of Ekurhuleni Metropolitan Municipality. Fifteen day-care centres were randomly selected in each sub-district region for measurement of lead concentrations. A minimum of 10 objects (wooden and plastic toys, chairs, walls, crayons and outdoor playing equipment) were randomly sampled in each day-care centre, and 450 objects were sampled. Children were randomly selected in each preschool, to ensure equal opportunity for all to be selected as participants. The population sample size was determined using $G^{*}$ Power statistical software (V. 3.0.10) $\quad(\alpha=0.05, \mathrm{OR}=0.3$ and $\beta=0.08)$; the expected sample size for a simple random sample and considering the type of analysis is 312.

\section{Recruitment}

After obtaining ethical clearance and permission from the relevant authorities, we explained the aim and the objectives of the project and the research activities to the participants. We also informed them of their rights (if they agreed to take part in the study), and we explained the risks and benefits of participation in the study. We then gave the parents or legal guardians an information letter and consent form written in their preferred language (English, isiZulu or Sepedi). They were given 7 days to return the forms. Once the parents gave parental consent, we obtained assent from the children on the day when blood samples were collected. 


\section{Data gathering}

Data were collected using three tools: an X-ray fluorescence (XRF) analyser, a LeadCare II analyser and a questionnaire. We appointed qualified nurses for the blood sampling, and we sought the assistance of a certified XRF technologist for environmental sampling.

\section{Measuring of lead concentrations in objects (tool 1)}

The XRF analyser was used to measure the concentrations of lead on painted surfaces (walls, equipment in the playground and so on), toys, furniture and other objects. The instrument detects the amount of lead in substances, by exposing the object to high-energy radiation, which causes lead to release X-rays at a frequency. Analysis of each object takes about $60 \mathrm{~s}$. Testing of the objects was done by the researchers in the absence of children and other personnel, to avoid exposing them to radiation. The operator was protected from radiation by a cover in front of the X-ray machine. The instrument was chosen because it has previously been used in local occupational and environmental settings. ${ }^{26-28} 31$

\section{Blood sampling (tool 2)}

Blood samples were taken in all the day-care centres that were sampled for environmental sampling. A professional trained and experienced nurse registered with the South African Nursing Council (SANC) took the blood samples from the children while they were seated on a portable medical bed. The children's hands were washed with water and soap and were dried with a chemical-free paper towel. Before the left-hand middle finger was pricked to collect venous blood, the fingertip was prep-swabbed using an alcohol wet wipe. Once the tip of the finger was pricked, two drops $(50 \mu \mathrm{L})$ of blood were collected and transferred using a sterile tube. The collected blood was analysed on site within $3 \mathrm{~min}$ using a portable LeadCare II analyser with a detection range from $3.3 \mu \mathrm{g} / \mathrm{dL}$ to $65 \mu \mathrm{g} /$ dL. The LeadCare II analyser uses an electroanalytical technique (the anodic stripping voltammetry method) to detect lead levels in the blood, and it is accepted by WHO. The LeadCare II kits have been validated in previous studies. $^{32} 33$ The parents of children with BLLs equal to or above $5 \mu \mathrm{g} / \mathrm{dL}$ (the minimum level where the action should be taken, as per Center for Disease Control and Prevention (CDC) standards) were referred to their nearest primary healthcare facility for further clinical management.

\section{Questionnaire (tool 3)}

The questionnaire was adapted from previous studies to meet the purpose of the current study. ${ }^{34-36}$ The information sheet and the questionnaire were translated into the two dominant local languages (isiZulu and Sepedi). We used the questionnaire to collect information on the children's age, gender, weight, height, diet (use of supplements such as iron and calcium) and health status (clinical symptoms); the household income status; the parents' marital status and the parents' or the guardians' educational levels and occupational status. It also included information on possible household lead exposure sources (home ownership, age, type, most fuel used, dwelling condition, water plumbing, use of herbal remedies, ownership of pets, proximity to mine dumps and industries and running a business at home), children's behavioural risks (hand-to-mouth habits, hand-washing practices, playing areas and outdoor and indoor activities) and parental risk factors (hobbies and smoking).

The self-administered questionnaires were given to the parents or legal guardians of the participants. The parents or guardians completed the questionnaires themselves at their home and returned them in a sealed nameless envelope to the day-care centre the following day. Completed questionnaires were collected by the researchers from the day-care centres. These were checked for completeness, and if there were any that were incomplete, the researchers contacted the participants' parents or legal guardians.

\section{DATA STORAGE AND MANAGEMENT}

Data collected (hard copies, eg, questionnaires and forms) were kept under lock and key. Soft copies were saved in the cloud and are accessible using a password. Only the research team members know the password. The data will be kept at the university for 10 years.

\section{Quality control}

The environmental samples were collected after the XRF analyser had been calibrated to ensure correct reading. The blood samples were taken by a qualified nurse with active registration with the SANC. The LeadCare II machine was calibrated for each new kit used. To collect information on risk factors, we used a questionnaire which was adapted from previous studies conducted in South Africa and elsewhere. ${ }^{2529}$ Only the questionnaire was piloted prior to the actual study, and we sought assistance from a biostatistician from the inception stage.

\section{Data analysis}

The data from the questionnaires, the results for the BLLs and the results for the surface and the object lead concentrations were entered into the Microsoft Excel spreadsheet for coding and data-cleaning purposes. It was then imported into the IBM SPSS V. 26 for analysis. Descriptive analysis was then done, where means, medians and IQRs were calculated for all continuous variables, while categorical variables were presented as percentages. In order to assess the relationships between the individual independent variables (sociodemographic variables and environmental and behavioural risk factors) and BLLs (the dependent variable), both univariable and multivariable logistic and linear regression models were fitted. Our model was built using the stepwise method, where a liberal $p$ value of 0.1 was used in selected variables, and then a backwards or maximum 
likelihood ratio test for the inclusion criteria of variables is employed to select variables significant at the $95 \%$ CI for the final model (multivariable regression). Estimates for the logistic regression model were presented as ORs. For the logistic regression, BLLs were recoded into two categories based on CDC criteria (high: $\geq 5 \mu \mathrm{g} / \mathrm{dL}$; low: $\leq 5 \mu \mathrm{g} / \mathrm{dL}$ ). The Hosmer-Lemeshow goodness-of-fit postestimation test is used to assess the model's fitness for the multivariable logistic regression model. Where necessary, Pearson's $\chi^{2}$ test, Fisher's exact test, the proportionality test, the two-sample Student's t-test and analysis of variance test are used to assess associations. Lastly, in the case where a continuous variable does not meet the assumptions for t-tests, a non-parametric equivalent of the t-test is employed.

\section{Ethics and dissemination}

The study was registered (NHRD no. 201801_041) on the National Health Research Database (NHRD), and it was granted ethical clearance (REC-01-141-2017) for nontherapeutic research involving minors from the Faculty of Health Sciences Research Ethics Committee (REC) of the University of Johannesburg in 2017. The ethical clearance was noted by the Gauteng Provincial Health Research Committee and the Ekurhuleni Metropolitan Municipality Research Ethics Committee. Permission was granted by the Ekurhuleni Metropolitan Municipality Department of Social Development to approach daycare centres. We further received permission from the owners or the management of the day-care centres. We then obtained parental or legal guardian consent prior to approaching the children. The children gave assent by providing a palm print to indicate their assent before taking part in the study. Children who cried, shook their heads or pulled their arm away during the drawing of blood or at any stage were excluded from the study, even though their parents or guardians had given consent for their participation.

The study sought to generate information on the risks associated with elevated BLLs among preschool children in the vulnerable group (2-5 years), in order to promote public health action to prevent long-term exposure to low to high lead levels in day-care centres. This action will encourage preschools to adopt or provide a lead-free environment. The researchers intend to communicate the results of the study to stakeholders at different platforms, including parent meetings, day-care centre board or management meetings and other forums. Lastly, they intend to present a paper at a regional or a national conference and to publish the results in an accredited journal.

\section{DISCUSSION}

BLLs below $2 \mu \mathrm{g} / \mathrm{dL}$ have been shown to have a negative health impact on children. ${ }^{37}$ Yet, children's playing environment is still highly contaminated with lead. A study in Gauteng Province showed that painted children's playing equipment contained lead levels of $10.4 \mathrm{mg} / \mathrm{cm}^{2}$, which was higher than the international reference $\left(1 \mathrm{mg} / \mathrm{cm}^{2}\right)$ level. ${ }^{26}$ Yet, environmental lead exposure is preventable, especially in children's environment. This study will assist in highlighting the need for a lead-free environment in preschools, and in advocating for such an environment.

Most studies in South Africa have focused on prenatal exposure and exposure to children older than 5 years, teenagers and adults. ${ }^{18} 323538-40$ These studies have associated elevated BLLs with personal, parental, residential and community risk factors. The strength of this study is that for the first time in South Africa it will be determined what the lead levels are and where do children study and spend most of their time. It will also determine the influence of environmental lead exposure in day-care centres on BLLs in children under 5 years.

Developed countries have introduced public intervention for surveillance and prevention of lead exposure at an early age in children. In 2006, South Africa banned the use of leaded petrol. Even though there was a decline in BLLs after this intervention, South African children aged 5 years or older and adolescents still had BLLs higher than $5 \mu \mathrm{g} / \mathrm{dL}$. Children aged 5-12 years from South African cities had BLLs ranging from $0.8 \mu \mathrm{g} / \mathrm{dL}$ to $32.3 \mu \mathrm{g} / \mathrm{dL},{ }^{36}$ while adolescents aged 13 years old were found to have BLLs ranging from $1.0 \mu \mathrm{g} / \mathrm{dL}$ to $28 \mu \mathrm{g} / \mathrm{dL}^{18}$

Preventing early exposure to lead and screening for BLLs can help to prevent the development of antisocial behaviour and poor academic performance among children, and it can prevent developmental complications and other negative health outcomes associated with environmental lead contamination among children. This study highlights the importance of implementing a health screening programme for lead, as well as the importance of creating and maintaining a lead-free zone for children, as recommended by WHO. ${ }^{41}$

A possible limitation of the study is that it focuses on lead exposure at the day-care centres, and it relies on the questionnaire to assess possible exposure at home. Therefore, the study results might not be generalised to other areas of the country due to different lead sources. Lastly, the study focuses on current (1 month or less) BLLs.

Acknowledgements The researchers would like to acknowledge Dr Vusi Nkosi for assisting with determining sampling size and advising on a data analysis plan. We would also like to thank professor Angela Mathee and Mrs Mirriam Mogotsi for providing the XRF machine and for measuring the lead concentrations in objects, respectively.

Contributors Developing and drafting of original study protocol: MC; drafting final manuscript: MC, TPM and NN; methodology: MC, TPM and NN. All the authors read and approved the final version.

Funding This research received no specific grant from any funding agency in the public, commercial or not-for-profit sectors. The study received funding from the University of Johannesburg Research Council (URC), URC Grant number: R6258829.

Competing interests None declared.

Patient and public involvement Patients and/or the public were not involved in the design, or conduct, or reporting, or dissemination plans of this research.

Patient consent for publication Not required.

Provenance and peer review Not commissioned; externally peer reviewed. 
Open access This is an open access article distributed in accordance with the Creative Commons Attribution Non Commercial (CC BY-NC 4.0) license, which permits others to distribute, remix, adapt, build upon this work non-commercially, and license their derivative works on different terms, provided the original work is properly cited, appropriate credit is given, any changes made indicated, and the use is non-commercial. See: http://creativecommons.org/licenses/by-nc/4.0/.

\section{ORCID iDs}

Thokozani Patrick Mbonane http://orcid.org/0000-0001-5802-4265

Nisha Naicker http://orcid.org/0000-0001-7223-7881

\section{REFERENCES}

1 Liu J, Ai Y, McCauley L, et al. Blood lead levels and associated sociodemographic factors among preschool children in the South eastern region of China. Paediatr Perinat Epidemiol 2012;26:61-9.

2 Kordas K, Ravenscroft J, Cao Y, et al. Lead exposure in low and middle-income countries: perspectives and lessons on patterns, Injustices, economics, and politics. Int J Environ Res Public Health 2018;15:2351.

3 Attina TM, Trasande L. Economic costs of childhood lead exposure in low- and middle-income countries. Environ Health Perspect 2013;121:1097-102.

4 Obeng-Gyasi E. Sources of lead exposure in various countries. Rev Environ Health 2019;34:25-34.

5 Harper CC, Mathee A, von Schirnding Y, et al. The health impact of environmental pollutants: a special focus on lead exposure in South Africa. Int J Hyg Environ Health 2003;206:315-22.

6 Mathee A, Street R. WITHDRAWN:Recycled Aluminium Cooking Pots: A Priority For The Unfinished Agenda Of Lead Exposure Reduction In Poorly Resourced Countries. Curr Opin Toxicol 2020.

7 Cossío-Torres P, Calderón J, Tellez-Rojo M, et al. Factors related to health outcomes and health risk behaviors of adolescents with lead exposure. A pilot study - Factores relacionados con el estado de salud y conductas de riesgo para la salud de adolescentes con exposición a plomo. Un estudio piloto. Salud Ment 2016;36:73.

8 Keramati MR, Manavifar L, Badiee Z, et al. Correlation between blood lead concentration and iron deficiency in Iranian children. Niger Med J 2013;54:325.

9 Hsueh Y-M, Lee C-Y, Chien S-N, et al. Association of blood heavy metals with developmental delays and health status in children. Sci Rep 2017;7:43608

10 Geier D, Kern J, Geier M. Blood lead levels and learning disabilities: a cross-sectional study of the 2003-2004 National health and nutrition examination survey (NHANES). Int J Environ Res Public Health 2017;14:1202-10.

11 Liu J, Liu X, Wang W, et al. Blood lead concentrations and children's behavioral and emotional problems: a cohort study. JAMA Pediatr 2014;168:737-45.

12 Olympio KPK, Oliveira PV, Naozuka J, et al. Surface dental enamel lead levels and antisocial behavior in Brazilian adolescents. Neurotoxicol Teratol 2010;32:273-9.

13 Blackowicz MJ, Hryhorczuk DO, Rankin KM, et al. The impact of lowlevel lead toxicity on school performance among Hispanic subgroups in the Chicago public schools. Int J Environ Res Public Health 2016;13.

14 Bellinger DC. Very low lead exposures and children's neurodevelopment. Curr Opin Pediatr 2008;20:172-7.

15 Haque M, Faruquee MH, Lahiry S, et al. Lead exposure and intellectual function: findings from primary school children in Bangladesh. Bangladesh J Med Sci 2012;11:292-7.

16 Needleman HL, McFarland C, Ness RB, et al. Bone lead levels in adjudicated delinquents. A case control study. Neurotoxicol Teratol 2002;24:711-7.

17 Nkomo P, Naicker N, Mathee A, et al. The association between environmental lead exposure with aggressive behavior, and dimensionality of direct and indirect aggression during midadolescence: birth to twenty plus cohort. Sci Total Environ 2018;612:472-9.
18 Nkomo P, Mathee A, Naicker N, et al. The association between elevated blood lead levels and violent behavior during late adolescence: the South African birth to twenty plus cohort. Environ Int 2017;109:136-45.

19 J. SAMPSON R, S. WINTER A. Poisoned development: assessing childhood lead exposure as a cause of crime in a birth cohort followed through adolescence. Criminology 2018:56:269-301.

20 McLaine P, Navas-Acien A, Lee R, et al. Elevated blood lead levels and reading readiness at the start of kindergarten. Pediatrics 2013;131:1081-9.

21 Prihartono NA, Djuwita R, Mahmud PB, et al. Prevalence of blood lead among children living in battery recycling communities in greater Jakarta, Indonesia. Int J Environ Res Public Health 2019;16:1276.

22 Mbongwe B, Barnes B, Tshabang J, et al. Exposure to lead among children aged 1-6 years in the city of Gaborone. Botswana J Env Heal Res 2010;10:17-26.

23 Tirima S, Bartrem C, von Lindern I, et al. Food contamination as a pathway for lead exposure in children during the 2010-2013 lead poisoning epidemic in Zamfara, Nigeria. Journal of Environmental Sciences 2018;67:260-72.

24 Amankwaa EF, Adovor Tsikudo KA, Bowman JA. 'Away' is a place: the impact of electronic waste recycling on blood lead levels in Ghana. Sci Total Environ 2017;601-602:1566-74.

25 Cao J, Li M, Wang Y, et al. Environmental lead exposure among preschool children in Shanghai, China: blood lead levels and risk factors. PLoS One 2014;9:e113297.

26 Mathee A, Singh E, Mogotsi M, et al. Lead-based paint on playground equipment in public children's parks in Johannesburg, Tshwane and Ekurhuleni. South African Med J = Suid 2009;99:819-21.

27 Kootbodien T, Mathee A, Naicker N, et al. Heavy metal contamination in a school vegetable garden in Johannesburg. South African Med J 2012;102:226-7.

28 Mathee A, Kootbodien T, Kapwata T, et al. Concentrations of arsenic and lead in residential garden soil from four Johannesburg neighborhoods. Environ Res 2018;167:524-7.

29 Mathee A, von Schirnding Y, Montgomery M, et al. Lead poisoning in South African children: the hazard is at home. Rev Environ Health 2005;19:347-61.

30 Canfield RL, Jusko TA, Kordas K. Environmental lead exposure and children's cognitive function. Ital J Pediatr 2005;31:293-300.

31 Gorce J-P, Roff M. Immediate screening of lead exposure in the workplace using portable X-ray fluorescence. J Occup Environ Hyg 2016;13:102-11.

32 Naicker $\mathrm{N}$, de Jager $\mathrm{P}$, Naidoo $\mathrm{S}$, et al. Is there a relationship between lead exposure and aggressive behavior in shooters? Int $J$ Environ Res Public Health 2018;15:1427.

33 Mathee A, de Jager P, Naidoo S, et al. Exposure to lead in South African shooting ranges. Environ Res 2017;153:93-8.

34 Mathee A. Blood Lead Levels in First Grade South African Children a Geographic \& Temporal Analysis, 2005. Available: https://core.ac. uk/reader/39665499

35 Mathee A, Röllin H, von Schirnding Y, et al. Reductions in blood lead levels among school children following the introduction of unleaded petrol in South Africa. Environ Res 2006;100:319-22.

36 Naicker N, Mathee A, Barnes B. A follow-up cross-sectional study of environmental lead exposure in early childhood in urban South Africa. S Afr Med J 2013;103:935-8.

37 Paulson JA, Brown MJ. The CDC blood lead reference value for children: time for a change. Environ Health 2019;18:16.

38 Naicker N, Norris SA, Mathee A, et al. Prenatal and adolescent blood lead levels in South Africa: Child, maternal and household risk factors in the birth to twenty cohort. Environ Res 2010;110:355-62.

39 von Schirnding Y, Mathee A, Kibel M, et al. A study of pediatric blood lead levels in a lead mining area in South Africa. Environ Res 2003;93:259-63.

40 Mathee A, Khan T, Naicker N, et al. Lead exposure in young school children in South African subsistence fishing communities. Environ Res 2013;126:179-83.

41 Ettinger AS, Leonard ML, Mason J. Cdc's lead poisoning prevention program: a long-standing responsibility and commitment to protect children from lead exposure. J Public Health Manag Pract 2019;25 Suppl 1, Lead Poisoning Prevention:S5-12. 